\title{
A THEOREM IN HOMOLOGICAL ALGEBRA AND STABLE HOMOTOPY OF PROJECTIVE SPACES
}

\author{
BY \\ ARUNAS LIULEVICIUS(1)
}

Introduction. The paper exhibits a general change of rings theorem in homological algebra and shows how it enables to systematize the computation of the stable homotopy of projective spaces.

Chapter I considers the following situation: $R$ and $S$ are rings with unit, $h: R \rightarrow S$ is a ring homomorphism, $M$ is a left $S$-module. If an $S$-free resolution of $M$ and an $R$-free resolution of $S$ are given, Theorem I.1. shows how to construct an $R$-free resolution of $M$.

Chapter II is devoted to computing the initial stable homotopy groups of projective spaces. Here the results of Chapter I are applied to the homomorphism $\alpha: A \rightarrow A$ of the Steenrod algebra over $Z_{2}$ (see I.3). The main tool in computing stable homotopy is the Adams spectral sequence [1]. Let $R P^{\infty}, C P^{\infty}, H P^{\infty}$ be the real, complex, and quaternionic infinite-dimensional projective spaces, respectively. If $X$ is a space, let $\Pi_{m}^{S}(X)$ denote the $m$ th stable homotopy group of $X$ [1]. Part of the results of Chapter II can be presented as follows:

$\begin{array}{lllllllcc}m: & 1 & 2 & 3 & 4 & 5 & 6 & 7 & 8 \\ R P^{\infty}: & Z_{2} & Z_{2} & Z_{8} & Z_{2} & 0 & Z_{2} & Z_{16} \oplus Z_{2} & Z_{2} \oplus Z_{2} \oplus Z_{2} \\ C P^{\infty}: & 0 & Z & 0 & Z & Z_{2} & Z & Z_{2} & Z \oplus Z_{2} \\ H P^{\infty}: & 0 & 0 & 0 & Z & Z_{2} & Z_{2} & 0 & Z\end{array}$

\section{Chapter I. Homological Algebra}

1. A change of rings theorem. Let $R$ and $S$ be rings with unit, $h: R \rightarrow S$ a homomorphism of rings; under $h$, any left $S$-module can be considered as a left $R$-module.

Let $M$ be a left $S$-module. Let $Y$ be an $S$-free resolution of $M: Y=\Sigma_{q \geqq 0} Y_{q}$, with differential $d^{\prime}$ and augmentation $\varepsilon^{\prime}$. Let $X_{q}$ be an $R$-free resolution of $Y_{q}$ : differential $d^{\prime \prime}$ and augmentation $\varepsilon_{q}$ onto $Y_{q}$.

Let $C=\Sigma_{q \geqq 0} X_{q}, C_{k}=\Sigma_{q+r=k} X_{q r}$, and augmentation $\varepsilon=\varepsilon^{\prime}\left(\Sigma_{q} \varepsilon_{q}\right)$. If

Presented to the Society, April 14, 1962; received by the editors April 5, 1962 and,in revised form, June 12, 1962.

(1) This paper was written while the author held a National Science Foundation postdoctoral fellowship. 
$f: C \rightarrow C$ is a homomorphism which lowers total degree, then $f=\Sigma_{k=0}^{\infty} f_{k}$, where $f_{k}: X_{q} \rightarrow X_{q-k}$.

TheOREM I.1. There exists a differential $d: C \rightarrow C$ such that $\{C, d, \varepsilon\}$ is an $R$-free resolution of $M$. The differential $d$ can be chosen to have the properties:

(1) $d_{0}$ is induced from $d^{\prime \prime}$,

(2) $d^{\prime} \varepsilon_{q+1}=\varepsilon_{q} d_{1}$,

(3) $\sum_{i=0}^{k} d_{i} d_{k-i}=0$;

conversely, any map with properties (1), (2), (3) is a differential which makes $C$ acyclic.

REMARK. Let $G$ be a finite group, $H$ a normal subgroup of $G, K$ a ring; let $R=K[G], S=K[G / H], M=K$. Theorem I.1 was proved by Wall [14] in this special case. The proof presented here is a straightforward translation to the general case.

Proof of Theorem I.1. Let us show that any $d$ with properties (1), (2), (3) makes $C$ acyclic. Filter $C$ by $F^{p} C=\Sigma_{q \leqq p} X_{q}$. The differential $d$ preserves filtration, and the associated spectral sequence converges to $H(C)$. The differential in $E^{0}$ is precisely $d_{0}$, hence $E^{1}=Y$, with $d^{1}$ corresponding to $d^{\prime}$ because of (2). Since $Y$ is a resolution of $M, E^{2}=E^{\infty}=M$, hence $C$ is acyclic.

To prove that $d$ with properties (1), (2), (3) exists is easy. Since the $X_{k}$ are $R$-free resolutions of $Y_{k}$, we can construct an $R$-map $d_{1}: X_{q, r} \rightarrow X_{q-1, r}$ such that $\varepsilon_{q-1} d_{1}=d^{\prime} \varepsilon_{q}$. To construct the maps $d_{k}, k \geqq 2$, we use induction on the total degree $q+r$ of $X_{q, r}$. We set $d_{k}=0$ if it lands in $X_{q^{\prime}, r^{\prime}}$ with $q^{\prime}<0$. Suppose $d$ has been defined on $X_{q^{\prime}, r}$, with $q^{\prime}+r^{\prime}<q+r$, and $d_{0}, \cdots, d_{k}$ have been defined on $X_{q, r}$. Let $f=-\sum_{i=1}^{k} d_{i} d_{k+1-i}$. We claim there exists a map $d_{k+1}$ such that $d_{0} d_{k+1}=f$. To prove this it suffices to prove that $d_{0} f=0$ and $\varepsilon_{q-k-1} f=0$, but this is easy:

$$
\begin{aligned}
d_{0} f & =-\sum_{i=1}^{k+1} d_{0} d_{i} d_{k+1-i}=\sum_{i=1}^{k+1} \sum_{j=1}^{i} d_{j} d_{i-j} d_{k+1-i} \\
& =\sum_{j=1}^{k+1} d_{j} \sum_{i=j}^{k+1} d_{i-j} d_{k+1-i}=0,
\end{aligned}
$$

which completes the proof of Theorem I.1.

2. Hopf algebras. Let $E, F$ be graded, connected, associative Hopf algebras over field a $K[12]$. Suppose that $F$ is a Hopf subalgebra of $E$. Then, according to Theorem 2.5 of [12], $E$ is free as a right (or left) $F$-module. Therefore we have

Proposition I.2. $E \otimes_{F}$ is an exact functor of left F-modules into left E-modules.

We shall say that $F$ is normal in $E$ if $F E=E F$, where $F$ denotes the augmentation ideal of $F$. Let $B=E / / F=E / E F$. 
Proposition I.3. If $W$ is an $F$-free resolution of $K$, then $E \otimes_{F} W$ is an $E$-free resolution of $B$.

Proof. Proposition I.2 and $E \otimes_{F} K=B$.

REMARK. Let $R=E, S=B$, and $h: R \rightarrow S$ the projection map. Let $M$ be a $B$ module, $Y=B \otimes \bar{Y}$ a $B$-free resolution of $M, U=F \otimes \bar{U}$ an $F$-free resolution of $K$. Then, according to the proposition above, we can take for $X_{q}$ in Theorem I.1. the complex $E \otimes \bar{Y}_{q} \otimes \bar{U}$ with the differential induced from $U$ (see [10]).

3. The Steenrod algebra. Let $A$ be the Steenrod algebra [11] over $Z_{2}$. The graded dual $A^{*}$ is a polynomial algebra and the squaring map in $A^{*}$ is a Hopf algebra map $\alpha^{*}$. Let $\alpha: A \rightarrow A$ be the dual of $\alpha^{*} ; \alpha$ is defined by $\alpha\left(S q^{2^{r+1}}\right)=S q^{2^{r}}$.

If $I$ is a finitely nonzero sequence of non-negative integers, then we let $S q^{I}$ denote the Milnor basis element corresponding to $I$. Let $\Delta_{i}$ be the sequence consisting of 1 in the $i$ th place and zeros elsewhere. Define the elements

$$
Q_{i}=S q^{\Delta_{i}}, \quad R_{i}=S q^{2 \Delta i}
$$

Let $C$ be the subalgebra of $A$ generated by 1 and $Q_{k}, k=0,1, \cdots ; B$ the subalgebra of $A$ generated by $1, Q_{0}$, and $R_{k}, k=0,1, \cdots$.

Proposition I.4. B and $C$ are normal Hopf subalgebras of $A$, and

$$
\begin{aligned}
& \text { Kernel } \alpha=A \bar{C}, \\
& \text { Kernel } \alpha \circ \alpha=A \bar{B} .
\end{aligned}
$$

Proof. Immediate consequence of Lemma 2.4.2 of [2].

REMARKS. 1. The preceding proposition states that we may consider $\alpha$ and $\alpha \circ \alpha$ as the projection maps $A \rightarrow A / / C, A \rightarrow A / / B$, respectively.

2. The map $\alpha$ halves the grading. Let $\tilde{A}$ denote $A$ with the grading of every element multiplied by two. Then $\alpha: A \rightarrow \tilde{A}$ preserves grading. The reader is asked to make such adjustments in the following pages.

It will be necessary to know the groups $\operatorname{Ext}_{C}^{s, t}\left(Z_{2}, Z_{2}\right), \operatorname{Ext}_{B}^{s, t}\left(Z_{2}, Z_{2}\right)$. The first is easily determined, for $C$ is a Grassman algebra:

$$
\operatorname{Ext}_{c}^{* * *}\left(Z_{2}, Z_{2}\right)=Z_{2}\left[q_{0}, \cdots, q_{k}, \cdots\right]
$$

where the polynomial generator $q_{k} \in \mathrm{Ext}^{1,2^{k+1}-1}$.

We compute $\operatorname{Ext}_{B}^{s, t}\left(Z_{2}, Z_{2}\right)$ using Theorem I.1. We shall use the standard minimal resolution of $Z_{2}$ over $C$. Generators will be in one-to- one correspondence with finitely nonzero sequences of integers $I$ (the free $C$-generater corresponding to $I$ will be denoted by $[I])$. Let $I=\left(i_{0}, i_{1}, \cdots, i_{k}, \cdots\right)$, then degree $[I]$ 
$=\Sigma_{k} i_{k}$, grade $[I]=\Sigma_{k} i_{k}\left(2^{k+1}-1\right)$. The differential in the minimal resolution is defined by

$$
d[I]=\sum_{r=0}^{\infty} Q_{r}\left[I-\Delta_{r}\right],
$$

where we set $\left[I-\Delta_{r}\right]=0$ if $i_{r}=0$.

According to Proposition I.4, $\operatorname{Ker} \alpha \mid B=B \bar{C}$, and $C$ is normal in $B$. For the module $X_{i, j}$ in Theorem $I .1$ we take the free $B$-module on generators $[I] \otimes[J]$, where degree $[I]=i$, degree $[J]=j$, and grade $([I] \otimes[J])=2$ grade $[I]+$ grade $[J]$. The augmentation $\varepsilon_{i}$ is defined by $\varepsilon_{i}([I] \otimes[J])=0$ if degree $[J]>0$, $\varepsilon_{i}([I] \otimes[J])=[I]$ if degree $[J]=0$. Both $d_{0}$ and $d^{\prime}$ are defined by the formula for $\tilde{d}$ above. An easy induction on the degree of $[J]$ shows that we can define the maps $d_{k}$ for $k \geqq 1$ as follows :

$$
\begin{aligned}
d_{1}[I] \otimes[J]= & \sum_{k} R_{k}\left[I-\Delta_{k}\right] \otimes[J]+\sum_{k}\left(j_{k+1}+1\right)\left[I-\Delta_{k}\right] \otimes\left[J-\Delta_{0}+\Delta_{k+1}\right] \\
d_{2}[I] \otimes[J]= & \sum_{i}\left(j_{i+1}+1\right) Q_{0}\left[I-\Delta_{0}-\Delta_{i}\right] \otimes\left[J+\Delta_{i+1}\right], \\
d_{3}[I] \otimes[J]= & \sum_{k<t}\left(j_{k+1}+1\right)\left(j_{t+1}+1\right)\left[I-\Delta_{0}-\Delta_{k}-\Delta_{t}\right] \otimes\left[J+\Delta_{k+1}+\Delta_{t+1}\right] \\
& +\sum_{t}\left(\begin{array}{c}
j_{t+1}+2 \\
2
\end{array}\right)\left[I-\Delta_{0}-2 \Delta_{t}\right] \otimes\left[J+2 \Delta_{t+1}\right],
\end{aligned}
$$

$d_{n}=0$ for $n \geqq 4$.

Since we will only use the groups $\operatorname{Ext}_{B}^{s, t}\left(Z_{2}, Z_{2}\right)$ for $t-s<13$, it is sufficient to consider the generators $[I] \otimes[J]$ in the resolution for which $i_{k}=0$ for $k \geqq 2$, $j_{r}=0$ for $r \geqq 3$. Thus for $t-s<13 \mathrm{Ext}_{B}^{s, t}\left(Z_{2}, Z_{2}\right)$ is additively the homology of the bi-graded algebra $Z_{2}\left[x_{0}, x_{1}, y_{0}, y_{1}, y_{2}\right]$, where grade $\left(x_{i}\right)=2^{i+2}-2$, $\operatorname{grade}\left(y_{j}\right)=2^{j+1}-1$, degree $\left(x_{i}\right)=\operatorname{degree}\left(y_{j}\right)=1$, under the differential $\delta_{1}+\delta_{2}$, where $\delta_{1}$ is a derivation and

$$
\delta_{1}\left(x_{i}\right)=0, \delta_{1}\left(y_{0}\right)=0, \delta_{1}\left(y_{j}\right)=y_{0} x_{j-1} ;
$$

$\delta_{2}$ is a map of $Z_{2}\left[x_{0}, x_{1}, y_{0}\right]$-modules with

$$
\begin{aligned}
\delta_{2}\left(x_{i}\right)= & 0, \quad \delta_{2}\left(y_{0}\right)=0 \\
\delta_{2}\left(y_{1}^{m_{1}} y_{2}^{m_{2}}\right)= & m_{1} m_{2} x_{0}^{2} x_{1} y_{1}^{m_{1}-1} y_{2}^{m_{2}-1}+\left(\begin{array}{c}
m_{1} \\
2
\end{array}\right) x_{0}^{3} y_{1}^{m_{1}-2} y_{2}^{m_{2}} \\
& +\left(\begin{array}{c}
m_{2} \\
2
\end{array}\right) x_{0} x_{1}^{2} y_{1}^{m_{1}} y_{2}^{m_{2}-2}
\end{aligned}
$$

and $\delta_{1} \delta_{2}+\delta_{2} \delta_{1}=0$. We list some obvious cycles under $\delta_{1}+\delta_{2}$ in the following table, and give classes in Ext $B_{B_{1}}$ which they determine. $\left(B_{1}\right.$ is the subalgebra of $B$ generated by $Q_{0}, R_{0}, R_{1}$, and $\operatorname{Ext}_{B_{1}}^{s, t}\left(Z_{2}, Z_{2}\right) \cong \operatorname{Ext}_{B}^{s, t}\left(Z_{2}, Z_{2}\right)$ for $\left.t-s<13\right)^{\circ}$ 
TABLE

\begin{tabular}{cccc} 
Cycle & Degree & Grade & Class \\
\hline$y_{0}$ & 1 & 1 & $g_{0}$ \\
$x_{0}$ & 1 & 2 & $k_{0}$ \\
$x_{1}$ & 1 & 6 & $k_{1}$ \\
$x_{0} y_{2}+x_{1} y_{1}$ & 2 & 9 & $\gamma$ \\
$y_{0} y_{1}^{2}+x_{0}^{2} y_{1}$ & 3 & 7 & $\tau_{0}$ \\
$y_{0} y_{2}^{2}+x_{0} x_{1} y_{2}$ & 3 & 15 & $\tau_{1}$ \\
$y_{1}^{4}$ & 4 & 12 & $\omega_{1}$ \\
$y_{2}^{4}$ & 4 & 28 & $\omega_{2}$ \\
$y_{0} y_{1}^{2} y_{2}^{2}+x_{0}^{2} y_{1} y_{2}^{2}$ & 5 & 21 & $\tau_{01}$ \\
$+x_{0} x_{1} y_{1}^{2} y_{2}$ & & & \\
\hline
\end{tabular}

Proposition I.5. $\operatorname{Ext}_{B_{1}}^{s, t}\left(Z_{2}, Z_{2}\right)$ is generated as an algebra by the classes

$$
g_{0}, k_{0}, k_{1}, \gamma, \tau_{0}, \tau_{1}, \tau_{01}, \omega_{1}, \omega_{2} .
$$

Furthermore, it is a free $Z_{2}\left[\omega_{1}, \omega_{2}\right]$-module with the following monomials as generators:

$$
\begin{aligned}
& g_{0}^{n}, \quad g_{0}^{n} \tau_{0}, \quad g_{0}^{n} \tau_{1}, \quad g_{0}^{n} \tau_{01}, \quad n \geqq 0, \\
& k_{0}^{i} k_{1}^{j}, \quad 0 \leqq i \leqq 2, \quad 0 \leqq j \quad(\text { if } i>0, \text { then } j \leqq 1), \\
& k_{0}^{i} k_{1}^{j} \gamma, \quad k_{1}^{j} \gamma^{2}, \quad k_{1}^{j} \gamma^{3} .
\end{aligned}
$$

Proof. Find the homology under $\delta_{1}$, decompose the homology into a tensor product of standard complexes under $\delta_{2}$, and use the Künneth theorem over the ring $Z_{2}\left[x_{0}\right]$.

REMARK. Once $\operatorname{Ext}_{B_{1}}\left(Z_{2}, Z_{2}\right)$ is known, it is very easy to construct a minimal resolution for $Z_{2}$ over $B_{1}$. The task is left to the reader.

4. Operations of Ext and the Adams spectral sequence. Let $A$ be the Steenrod algebra over $Z_{p}, L$ a left $A$-module. There is a natural map

$$
\mu: \operatorname{Ext}_{A}^{q, u}\left(L, Z_{p}\right) \otimes \operatorname{Ext}_{A}^{r, v}\left(Z_{p}, Z_{p}\right) \rightarrow \operatorname{Ext}_{A}^{q+r, u+v}\left(L, Z_{p}\right)
$$

which makes $\operatorname{Ext}_{A}\left(L, Z_{p}\right)$ into a right $\operatorname{Ext}_{A}\left(Z_{p}, Z_{p}\right)$-module. For the definition of $\mu$ see, for example, [2]. We write $\alpha * \beta$ for $\mu(\alpha \otimes \beta)$. 
For the Adams spectral sequence see [1].

THEOREM I.6 (ADAMs). The spectral sequence for the sphere $S^{0}$ operates on the spectral sequence for any arbitrary space $X$. In particular, if

$$
h \in \operatorname{Ext}_{A}^{s, t}\left(Z_{p}, Z_{p}\right), \quad a \in \operatorname{Ext}_{A}^{u, v}\left(H^{*}(X), Z_{p}\right),
$$

and $d_{j}(h)=0, j=2, \cdots, r, d_{k}(a)=0, k=2, \cdots, r-1$, then

$$
d_{r}(\{a * h\})=\left\{d_{r} a\right\} * h .
$$

Proof. The proof of Theorem 2.2 of [1]; see also Théorème IIB, Exposé 19 of [6].

\section{CHAPTER II. STABLE HOMOTOPY OF PROJECTIVE SPACES}

1. The prime $p=2$. Let $R P^{\infty}, C P^{\infty}, H P^{\infty}$ be the real, complex, and quaternionic infinite-dimensional projective spaces, respectively. It is well known that

$$
\begin{aligned}
& H^{*}\left(R P^{\infty} ; Z_{2}\right)=Z_{2}[x], \\
& H^{*}\left(C P^{\infty} ; Z_{2}\right)=Z_{2}[y], \\
& H^{*}\left(H P^{\infty} ; Z_{2}\right)=Z_{2}[u],
\end{aligned}
$$

where $x, y, u$ are the nonzero $1,2,4$-dimensional classes, respectively. Let $L, M, N$ be the elements of positive degree in (1), (2), (3), in the order given. Let $\alpha: A \rightarrow A$ be the dual of the squaring map (see Proposition I.3).

Proposition II.1. There are $Z_{2}$-isomorphisms $f: M \rightarrow L, g: N \rightarrow M$ such that the following diagram is commutative:

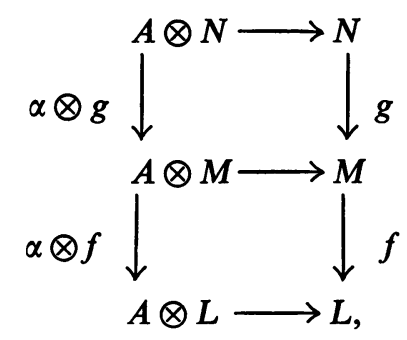

where the horizontal arrows indicate the action of $A$.

Proof. According to [11], if $\theta \in A$, then

$$
\theta x=\sum_{n=0}^{\infty}\left\langle\xi_{n}, \theta\right\rangle x^{2^{n}} .
$$

Let $h: R P^{\infty} \rightarrow C P^{\infty}$ be a map such that $h^{*}(y)=x^{2} ; h^{*}$ is a monomorphism. Thus from (5) and $h^{*}$ 


$$
\theta y=\sum_{n=0}^{\infty}\left\langle\xi_{n}^{2}, \theta\right\rangle y^{2^{n}} .
$$

Let $f: M \rightarrow L$ be the algebra map given by $f(y)=x$. Then $f(\theta y)=\alpha(\theta) f(y)$, for $\left\langle\xi_{n}^{2}, \theta\right\rangle=\left\langle\alpha^{*}\left(\xi_{n}\right), \theta\right\rangle=\left\langle\xi_{n}, \alpha(\theta)\right\rangle$. With this choice for $f$, the bottom rectangle of (4) is commutative. The proof is completed by defining $g(u)=y$ and considering a map $k: C P^{\infty} \rightarrow H P^{\infty}$ such that $k^{*}(u)=y^{2}$.

RemarK. Proposition II.1 is used by S. P. Novikov in his investigation of Thom spectra (dissertation - unpublished).

According to the proposition $M$ and $N$ are isomorphic to $L$ as $A$-modules through the homomorphisms $\alpha, \alpha \circ \alpha$, respectively. We are all set to apply the change of rings Theorem I.1. since we know the cohomology of the subalgebras $C$ and $B$ (at least in low dimensions, see Proposition I.3).

Before we introduce the results, let us define some elements in

$$
\operatorname{Ext}_{A}\left(Z_{2} Z_{2}\right): g_{0} \in \operatorname{Ext}^{1,1}, \quad h_{i} \in \operatorname{Ext}^{1,2^{i+1}}, \quad i=0,1, \cdots
$$

(the element $g_{0}$ corresponds to the element $h_{0}$ of [2]; our $h_{i}$ corresponds to $h_{t+1}$ of [2]).

Proposition II.2. As an $\operatorname{Ext}_{A}\left(Z_{2}, Z_{2}\right) \in \operatorname{module}$, $\operatorname{Ext}_{A}{ }^{s, t}\left(L, Z_{2}\right)$ has the following elements as generators for $t-s \leqq 10$ (if $s \leqq 2$ ) and $t-s \leqq 9$ (if $s>2$ ):

$$
e_{0,1}, e_{0,3}, e_{0}, e_{2,10}, e_{4,13}
$$

where $e_{s, t}$ denotes a nontrivial class in $\operatorname{Ext}_{A}^{\mathrm{s}, t}\left(L, Z_{2}\right) . A Z_{2}$-basis in these dimensions is given by the following set of classes:

$$
\begin{aligned}
& e_{0,1}, \quad e_{0,1} * h_{0}, \quad e_{01} * h_{1}, \quad e_{0,1} * h_{2}, \quad e_{01} * h_{0} h_{2}, \\
& e_{0,1} * h_{1}^{2}, \quad e_{0,3}, \quad e_{0,3} * g_{0}, \quad e_{0,3} * g_{0}^{2}, \quad e_{0,3} * h_{1}, \\
& e_{0.3} * h_{2}, \quad e_{03} * g_{0} h_{2}, \quad e_{0,7} * g_{0}^{k}, \quad k=0,1,2,3, \\
& e_{0.7} * h_{0}, \quad e_{0,7} * h_{0}^{2}, \quad e_{2,10}, \quad e_{2,10} * h_{0}, \quad e_{4,13} .
\end{aligned}
$$

Proof. Explicit minimal resolution, using the methods of [8].

Remarks. Compare Proposition II.2 with the results of Adams vanishing Theorem [4]. Also $e_{4,13}=P e_{0}$ (see Theorem 5 of [4]).

Proposition II.3. $\operatorname{Ext}_{\boldsymbol{A}}^{s, t}\left(M, Z_{2}\right)$ has the following $Z_{2}$-basis for $t-s \leqq 11$ :

$$
\begin{aligned}
& e_{02} * g_{0}^{n}, \quad e_{0,6} * g_{0}^{n}, \quad e_{15} * g_{0}^{n}, \quad e_{212} * g_{0}^{n}, \quad e_{3,11} * g_{0}^{n}, \quad n=0,1, \cdots, \\
& e_{0,2} * h_{1}, \quad e_{0,2} * g_{0} h_{1}, \quad e_{0,2} * h_{2} g_{0}^{k}, \quad k=0,1,2,3, \\
& e_{0,6} * h_{0}, \quad e_{06} * h_{0}^{2}, \quad e_{2,13} * g_{0}^{k}, \quad k=0,1,2,3, \quad e_{3,14} .
\end{aligned}
$$

Proposition II.4. $\operatorname{Ext}_{A}^{s, t}\left(N, Z_{2}\right)$ for $t-s \leqq 13$ has the following $Z_{2}$-basis: 


$$
\begin{aligned}
& e_{0,4} * g_{0}^{n}, \quad e_{0,12} * g_{0}^{n}, \quad e_{3,11} * g_{0}^{n}, \quad n=0,1,2, \cdots, \\
& e_{0,4} * h_{0}, \quad e_{0,4} * h_{0}^{2}, \quad e_{1,10}, \\
& e_{1,10} * h_{0}, \quad e_{1,10} * h_{0}^{2}, \quad e_{1,12} * g_{0}^{k}, \quad k=0,1,2,3, \\
& e_{1,12} * h_{0}, \quad e_{213}, \quad e_{2,13} * h_{0}, \quad e_{2,13} * h_{0}^{2}, \quad e_{5,18}, \quad e_{0,12} * h_{0} .
\end{aligned}
$$

Proposition II.3 and II.4 are proved by using the constructions of Theorem I.1. In the proof of Proposition II.3 we take an $A$-minimal resolution $Y$ of $L$ and take the tensor product of $Y$ with a minimal resolution of $Z_{2}$ over $C$. In the proof of Proposition II.4 the tensor product of $Y$ with a minimal resolution of $Z_{2}$ over $B$ is examined. In both cases, for the range of $s$ and $t$ given, only the map $d_{1}$ need be examined.

We give a sample computation. The minimal resolution of $L$ over $A$ for $t-s \leqq 5$ can be taken as follows:

$$
0 \leftarrow L \stackrel{\varepsilon}{\leftarrow} C_{0} \stackrel{d}{\leftarrow} C_{1} \stackrel{d}{\leftarrow} C_{2} \stackrel{d}{\leftarrow} 0 \leftarrow 0 \cdots,
$$

where $C_{0}$ is free on $c_{0,1}, c_{0,3}, C_{1}$ is free on $c_{1,3}, c_{1,4}, c_{1,5}, C_{2}$ is free on $c_{2,5}$; the maps $\varepsilon, d$ are defined to be

$$
\begin{aligned}
& \varepsilon\left(c_{0,1}\right)=x, \varepsilon\left(c_{0,3}\right)=x^{3} \\
& d\left(c_{1,3}\right)=a_{1} c_{0,1}, \\
& d\left(c_{1,4}\right)=Q_{0} c_{03}+Q_{1} c_{0,1}, \\
& d\left(c_{1,5}\right)=a_{2} c_{0,1}, \\
& d\left(c_{2,5}\right)=Q_{0} c_{1,4}+a_{1} c_{1.3},
\end{aligned}
$$

where $a_{i}=S q^{2 i}, Q_{i+1}=\left[a_{i+1}, Q_{i}\right]$.

Take generators $[I]$ of a minimal resolution $W$ of $Z_{2}$ over $C$ in one-to-one correspondence with finitely nonzero sequence $I$ of non-negative integers. We denote by $\Delta_{i}$ the sequence consisting of 1 in the $i$ th place and zeroes elsewhere; we let $I-J$ be the sequence of term-by-term differences (we set $[I-J]=0$ if at least one entry is negative). The differential $d^{\prime \prime}$ in $W$ is defined by

$$
d^{\prime \prime}[I]=\sum_{i=0}^{\infty} Q_{i}\left[I-\Delta_{i}\right]
$$

Let us show as an example that we can define $d_{1}$ on $c_{1,5} \otimes\left[n \Delta_{0}\right]$ as

$$
a_{3} c_{0,1} \otimes\left[n \Delta_{0}\right]+a_{1} a_{2} c_{01} \otimes\left[(n-1) \Delta_{0}+\Delta_{1}\right]+a_{1} c_{01} \otimes\left[(n-1) \Delta_{0}+\Delta_{2}\right]
$$

$$
\begin{aligned}
& +a_{2} c_{01} \otimes\left[(n-2) \Delta_{0}+2 \Delta_{1}\right]+c_{01} \otimes\left[(n-2) \Delta_{0}+\Delta_{1}+\Delta_{2}\right] \\
& +a_{1} c_{01} \otimes\left[(n-3) \Delta_{0}+3 \Delta_{1}\right]+c_{0,1} \otimes\left[(n-4) \Delta_{0}+4 \Delta_{1}\right] .
\end{aligned}
$$


We shall need relations in addition to those exhibited in Chapter I.3 :

$$
\begin{aligned}
& Q_{0} a_{3}=a_{3} Q_{0}+a_{1} a_{2} Q_{1}+a_{1} Q_{2}, \\
& Q_{0} a_{2}=a_{2} Q_{0}+a_{1} Q_{1} .
\end{aligned}
$$

The proof that (6) is admissible by induction on $n$. Since $\alpha\left(a_{3}\right)=a_{2},(6)$ is fine for $n=0$. Suppose (6) is acceptable for $n>0$ :

$$
\begin{aligned}
d_{1} d_{0}\left(c_{1,5} \otimes\left[(n+1) \Delta_{0}\right]\right)= & d_{1} Q_{0} c_{1,5} \otimes\left[n \Delta_{0}\right] \\
= & Q_{0} a_{3} c_{0,1} \otimes\left[n \Delta_{0}\right] \\
& +Q_{0} a_{1} a_{2} c_{0,1} \otimes\left[(n-1) \Delta_{0}+\Delta_{1}\right] \\
& +Q_{0} a_{1} c_{0,1} \otimes\left[(n-1) \Delta_{0}+\Delta_{2}\right] \\
& +Q_{0} a_{2} c_{0.1} \otimes\left[(n-2) \Delta_{0}+2 \Delta_{1}\right] \\
& +Q_{0} c_{0,1} \otimes\left[(n-2) \Delta_{0}+\Delta_{1}+\Delta_{2}\right] \\
& +Q_{0} a_{1} c_{0,1} \otimes\left[(n-3) \Delta_{0}+3 \Delta_{1}\right] \\
& +Q_{0} c_{0} \otimes\left[(n-4) \Delta_{0}+4 \Delta_{1}\right] \\
= & \left.a_{3} Q_{0}+a_{1} a_{2} Q_{1}+a_{1} Q_{2}\right) c_{0,1} \otimes\left[n \Delta_{0}\right] \\
& +\left(a_{1} a_{2} Q_{0}+a_{2} Q_{1}+Q_{2}\right) c_{0,1} \otimes\left[(n-1) \Delta_{0}+\Delta_{1}\right] \\
& +\left(a_{1} Q_{0}+Q_{1}\right) c_{0,1} \otimes\left[(n-1) \Delta_{0}+\Delta_{2}\right] \\
& +\left(a_{2} Q_{0}+a_{1} Q_{1}\right) c_{0,1} \otimes\left[(n-2) \Delta_{0}+2 \Delta_{1}\right] \\
& +Q_{0} c_{01} \otimes\left[(n-2) \Delta_{0}+\Delta_{1}+\Delta_{2}\right] \\
& +\left(a_{1} Q_{0}+Q_{1}\right) c_{0.1} \otimes\left[(n-3) \Delta_{0}+3 \Delta_{1}\right] \\
& +Q_{0} c_{0,1} \otimes\left[(n-4) \Delta_{0}+4 \Delta_{1}\right]
\end{aligned}
$$

which is precisely $d_{0}$ of (6) for $n+1$, which completes the inductive step.

Let $\Pi_{m}^{s}(X ; p)$ be the $m$ th stable homotopy group of $X$ [1] modulo the subgroup of elements having finite order prime to $p . \Pi_{m}^{S}(X ; p)$ may be computed up to extensions by the Adams spectral sequence for the prime $p$; the extension can often be determined if we remark that ${ }^{*} g_{0}$ corresponds to multiplication by $p$ in $\Pi_{*}^{S}$.

Proposition II.5. In the Adams spectral sequence $(p=2)$ for $R P^{\infty}$ all differentials vanish in total degrees $\leqq 10$.

Proof. Since in the Adams spectral sequence for the sphere $d_{r}\left(g_{0}\right)=d_{r}\left(h_{0}\right)$ $=d_{r}\left(h_{1}\right)=d_{r}\left(h_{2}\right)=0$ for all $r$ [4] according to Theorem I.6. it suffices to prove 
that all differentials vanish on $e_{01}, e_{0,3}, e_{07}, e_{210}, e_{413}$, but this is easy for the differentials land on groups which are zero according to Proposition II.2.

Since $R P^{\infty}=K\left(\mathrm{Z}_{2}, 1\right)$ we do not have to consider the spectral sequences for $p$ odd: they are all zero. Since $* g_{0}$ corresponds to multiplication by 2 we have:

THEOREM II.6. The stable homotopy groups $\Pi_{k}^{S}\left(R P^{\infty}\right)$ are as follows for $k \leqq 9$ :

$$
\begin{array}{ll}
k: & \Pi_{k}^{S}: \\
0 & 0 \\
1 & Z_{2} \\
2 & Z_{2} \\
3 & Z_{8} \\
4 & Z_{2} \\
5 & 0 \\
6 & Z_{2} \\
7 & Z_{16} \oplus Z_{2} \\
8 & Z_{2} \oplus Z_{2} \oplus Z_{2} \\
9 & Z_{2} \oplus Z_{2} \oplus Z_{2} \oplus Z_{2} .
\end{array}
$$

We precede the next theorem by a proposition about stable secondary cohomology operations.

Proposition II.7 (ADAMs). There exists a stable secondary cohomology operation $\Psi$ of degree 4 such that if $y \in H^{2}\left(C P^{\infty} ; Z_{2}\right)$ then $\Psi(y)$ is defined and

$$
\Psi(y)=y^{3} \text { modulo zero. }
$$

Proof. This is Theorem 4.4.1 of [2].

THEOREM II.8. In the Adams spectral sequence for $C P^{\infty}(p=2)$ the only nontrivial differential in total degrees $\leqq 9$ is

$$
d_{2}\left(e_{06}\right)=e_{02} * g_{0} h_{1} \text {. }
$$

Furthermore, the groups $\Pi_{m}^{S}\left(C P^{\infty} ; Z_{2}\right)$ are as follows for $m \leqq 8$ :

$$
\begin{array}{cccccccccc}
m: & 0 & 1 & 2 & 3 & 4 & 5 & 6 & 7 & 8 \\
\Pi_{m}^{S}: & 0 & 0 & Z & 0 & Z & Z_{2} & Z & Z_{2} & Z \oplus Z_{2} .
\end{array}
$$

Proof. Suppose $a * g_{0}^{j}=0$ for some $j$. Then if $d_{r}(a)=b, b * g_{0}^{j}=0$ in $E_{r}$, 
according to Theorem I.6. This settles all differentials in total degrees $\leqq 9$, except $d_{2}\left(e_{0,6}\right)$. According to Proposition II.7, $e_{0,6}$ cannot be a $d_{r}$-cycle for all $r$, since it is not in the image of the mod 2 Hurewicz homomorphism. This implies that $r=2$, for $d_{r}, r>2$ is automatically zero on $e_{0,6}$.

THEOREM II.9. In the Adams spectral sequence for $\operatorname{HP}^{\infty}(p=2)$ all differentials vanish in total degrees $\leqq 11$. Furthermore, the groups $\Pi_{m}^{S}\left(H P^{\infty} ; 2\right)$ for $m \leqq 10$ are as follows:

$\begin{array}{cccccccccccc}m: & 0 & 1 & 2 & 3 & 4 & 5 & 6 & 7 & 8 & 9 & 10 \\ \Pi_{m}^{S}: & 0 & 0 & 0 & 0 & Z & Z_{2} & Z_{2} & 0 & Z & Z_{2} & Z_{2} .\end{array}$

Proof. Proposition II.4 and argument as for Theorem II.8.

2. The primes $p>2$. In order to complete our study of the initial stable homotopy of projective spaces, we must examine the Adams spectral sequences for $C P^{\infty} H P^{\infty}$, for primes $p>2$.

The following two propositions are proved by constructing minimal resolutions for low total degrees. The task is straightforward and is left to the reader.

Let $M=\tilde{H}^{*}\left(C P^{\infty} ; Z_{p}\right)$ the augmented cohomology of $C P^{\infty}, p$ an odd prime, $A$ the Steenrod algebra over $Z_{p}$.

Proposition II.10. $A Z_{p}$-basis $(p>2)$ for $\operatorname{Ext}_{A}^{s, t}\left(M, Z_{p}\right)$ for $t-s \leqq 6 p-4$ is furnished by classes

$$
\begin{aligned}
& e_{0,2 j} * g_{0}^{n}, \quad e_{1,2 k+2 p-1} * g_{0}^{n}, \quad e_{2,2 r+4 p-2} * g_{0}^{n}, \\
& e_{14 p-2}, \quad e_{1,4 p-2} * g_{0},
\end{aligned}
$$

where $j=1, \cdots, p-1,2 p-1, k=1, \cdots, p-1, \quad r=2, \cdots, p-1 \quad(p>3$ for $r)$, $n=0,1, \cdots ;$ if $p=3$, we have in addition

$$
e_{0,2} * h_{1}, \quad e_{1,4 p-2} * h_{0}, \quad e_{0,2} * h_{1} g_{0}, \quad e_{0,2} * h_{1} g_{0}^{2} \text {. }
$$

Let $N=\tilde{H}^{*}\left(H P^{\infty} ; Z_{p}\right)$.

Proposition II.11. Let $p>2$. Then $\operatorname{Ext}_{A}^{s, t}\left(N, Z_{p}\right)$ for $t-s \leqq 6 p-2$ has the following elements as a $Z_{p}$-basis:

$$
\begin{array}{ll}
e_{0,4 k} * g_{0}^{n}, & e_{1,4 j+2 p-1} * g_{0}^{n}, \quad e_{2,4 j+4 p-2} * g_{0}^{n}, \\
e_{0.4} * h_{0}, & e_{04} * h_{0} g_{0}, \quad e_{04} * h_{0} g_{0}^{2},
\end{array}
$$

where $n=0,1, \cdots, k=1, \cdots, \frac{1}{2}(p-1), \frac{1}{2}(3 p-1), j=1, \cdots, \frac{1}{2}(p-1)$.

We are now ready to examine the Adams spectral sequence for $C P^{\infty}, H P^{\infty}$ for an odd prime $p$.

Proposition II.12. There exists a stable secondary cohomology operation $\Lambda$ of 
degree $4 p-4$, defined on cohomology classes $x$ such that $Q_{0} x=0, Q_{1} x=0$, $P^{2} x=0$, such that

$$
\Lambda(y)=b y^{2 p-1} \text { modulo zero, }
$$

where $b \neq 0$ and $y \in H^{2}\left(C P^{\infty} ; Z_{p}\right)$.

Proposition II.13. There exists a stable secondary cohomology operation $\Gamma$ of degree $6 p-6$ such that

(i) $\Gamma$ is defined on $y \in H^{2}\left(C P^{\infty} ; Z_{p}\right) u \in H^{4}\left(H P^{\infty} ; Z_{p}\right)$

(ii) $\Gamma(y)=c y^{3 p-2}$, modulo zero, where $c \neq 0$ in $Z_{p}$,

(iii) $\Gamma(u)=2 c u^{(3 p-1) / 2}$, modulo zero.

Propositions II.11 and II.12 are proved as in [9] using [2].

Proposition II.14. (i) The only nontrivial differential in the Adams spectral sequence for $C P^{\infty}$ and $p \geqq 5$ for total degree $\leqq 6 p-4$ is given by

where $b \neq 0$ in $Z_{p}$.

$$
d_{2}\left(e_{0,4 p-2}\right)=b e_{1,4 p-2} * g_{0}
$$

(ii) Statement (i) is valid for $p=3$ in total degrees $\leqq 13$.

Proof. Consider the case $p \geqq 5$. According to Proposition II.10 all nonzero elements of $\operatorname{Ext}_{w}\left(M, Z_{p}\right)$ have even total degree-except $e_{1,4 p-2}$ and $e_{1,4 p-2} * g_{0}$. The only elements in total degree $4 p-4$ are the basis elements $e_{1,2 p-2+2 p-1} * g_{0}^{n}$. Theorem I.6. shows that all differentials vanish on $e_{1,4 p-2}$ for $e_{1,4 p-2} * g_{0}^{2}=0$. In order to prove (i) it remains to show that the stable mod $p$ Hurewicz homomorphism is zero in dimension $4 p-2$. This is taken care of by Proposition II.12.

THEOREM II.15. (i) If $p \geqq 5$ the groups $\Pi_{k}^{S}\left(C P^{\infty} ; p\right)$ for $k \leqq 6 p-4$ are as follows:

$$
\begin{array}{ll}
\Pi_{k}^{S}\left(C P^{\infty} ; p\right)=Z & \text { if } k=2,1 \leqq i \leqq 3 p-2, \\
\Pi_{k}^{S}\left(C P^{\infty} ; p\right)=0 & \text { if } k=2 i+1, i \neq 2 p-2 \\
\Pi_{k}^{S}\left(C P^{\infty} ; p\right)=Z_{p} & \text { if } k=4 p-3
\end{array}
$$

(ii) the groups $\Pi_{k}^{S}\left(C P^{\infty} ; 3\right)$ for $k \leqq 12$ are as follows:

$$
\begin{array}{llllllllllll}
k: & 2 & 3 & 4 & 5 & 6 & 7 & 8 & 9 & 10 & 11 & 12 \\
\Pi_{k}^{S}: & Z & 0 & Z & 0 & Z & 0 & Z & Z_{3} & Z & 0 & Z \oplus Z_{3} .
\end{array}
$$

Proof. Propositions II.10, II.14.

Proposition II.16. In the Adams spectral sequence for $H P^{\infty}$ and $p \geqq 3$ the only nontrivial differential for total degrees $\leqq 6 p-2$ is

where $b \neq 0$ in $Z_{p}$.

$$
d_{2}\left(e_{0,6 p-2}\right)=b e_{0,4} * h_{0} g_{0},
$$


Proof. According to Proposition II.11 the only elements of odd total degree $\leqq 6 p-2$ are the classes $e_{0,4} * h_{0} g_{0}^{r}, \quad r=0,1,2$. All differentials on $e_{0,4}$ vanish, thus we only need to evaluate $d_{2}$ and $d_{3}$ on $e_{0,6 p-2}$. Proposition II.13 implies that one of these two differentials is nonzero on $e_{0,6 p-2}$. We use a folk theorem, which can be proved using the approach of [8] to the Adams spectral sequence: suppose a stable secondary cohomology operation corresponding to an element $u \in E_{2}^{2}$, has a minimal $A$-generator as image; suppose this generator determines the class $v \in E_{2}^{0, *}$ then $d_{2}(v)=u$. The proof is completed by remarking that the operation $\Gamma$ of Proposition II.13 corresponds to $e_{04} * h_{0} g_{0}$.

THEOREM II.17. If $p \geqq 3$, the groups $\Pi_{m}^{S}\left(H P^{\infty} ; p\right)$ for $m \leqq 6 p-2$ are as follows

$$
\begin{array}{ll}
\Pi_{4 k}^{S}\left(H P^{\infty} ; p\right)=Z & 0<4 k \leqq 6 p-2, \\
\Pi_{2 j-1}^{S}\left(H P^{\infty} ; p\right)=0 & 2 j-1 \leqq 6 p-2, j \neq 3 p-1, \\
\Pi_{6 p-3}^{S}\left(H P^{\infty} ; p\right)=Z_{p} . &
\end{array}
$$

Proof. Proposition II. 16.

\section{REFERENCES}

1. J. F. Adams, On the structure and applications of the Steenrod algebra, Comment. Math. Helv. 32 (1958), 180-214.

2. - On the non-existence of elements of Hopf invariant one, Ann. of Math. (2) 72 (1960), 20-104.

3. - A finiteness theorem in homological algebra, Proc. Cambridge Philos. Soc. 57 (1961), 31-36.

4. - Stable homotopy theory, (lecture notes), Berkeley, 1961.

5. A. Borel et J.-P. Serre, Groupes de Lie et puissances reduites de Steenrod, Amer. J. Math 75 (1953), 409-448.

6. Séminaire Henri Cartan, 11ème année: 1958/59: Invariant de Hopf et operations cohomologiques secondaires, Paris, 1959.

7. S. Eilenberg, Homological dimension and syzygies, Ann. of Math. (2) 64 (1956), 328-336.

8. H. Gershenson, Relationships between the Adams spectral sequence and Toda's calculations of the stable homotopy of spheres, Math. Z. 81 (1963), 229-259.

9. A. Liulevicius, The factorization of cyclic reduced powers by secondary cohomology operations, Mem. Amer. Math. Soc. No. 42 (1962), 113 pp.

10. - Homotopy of Thom spectra, Amer. J. Math. (to appear).

11. J. Milnor, The Steenrod algebra and its dual, Ann. of Math. (2) 67 (1958), 150-171.

12. J. Milnor and J. C. Moore, On the structure of Hopf algebras (to appear).

13. C. T. C. Wall, Generators and relations for the Steenrod algebra, Ann. of Math. (2) 72, (1960), 429-444.

14. —_ Resolutions for extensions of groups, Proc. Cambridge Philos. Soc. 57 (1961) 251-255.

The University of Chicago,

ChicAGo, Illinois

THe INSTITUTE FOR AdVANCEd Study,

Princeton, New JeRSEY 